\title{
Agradecimentos aos revisores
}

\section{Acknowledgments to IPecege Journal Reviewers}

A Revista IPecege agradece aos revisores ad hoc listados abaixo por seu excelente trabalho compartilhando seu conhecimento científico na avaliação criteriosa dos manuscritos, possibilitando a publicação de artigos de alto nível dentro dos rigores éticos. Suas colaborações são essenciais para esse periódico que adota o processo de revisão por pares, peer review.

\begin{tabular}{|c|c|c|}
\hline Revisores ad hoc 2017 & Instituição & UF/País \\
\hline Adolfo Fernandes & Instituto Federal do Acre & AC - Brasil \\
\hline Adriana Ferreira Silva & Universidade Federal de Goiás & GO - Brasil \\
\hline Adriana Sbardelotto Di Domenico & Universidade Tecnológica Federal do Paraná & PR - Brasil \\
\hline Alexandre de Vicente Ferraz & Instituto de Pesquisas e Estudos Florestais & SP - Brasil \\
\hline Alvaro Jose Periotto & Universidade Estadual de Maringá & PR - Brasil \\
\hline $\begin{array}{l}\text { Amanda Massaneira de Souza } \\
\text { Schuntzemberger }\end{array}$ & Universidade Estadual de Londrina & PR - Brasil \\
\hline Ana Paula Donofrio & $\begin{array}{l}\text { Centro Universitário do Instituto de Educação } \\
\text { Superior de Brasília }\end{array}$ & DF - Brasil \\
\hline André Brugnara Soares & Universidade Tecnologica Federal do Paraná & PR - Brasil \\
\hline Andre Luiz de Castro Leal & Universidade Federal Rural do Rio de Janeiro & RJ - Brasil \\
\hline Andrea Belem Costa & Universidade Federal do Amazonas & AM - Brasil \\
\hline Andreia Cristina de Oliveira Adami & $\begin{array}{l}\text { Centro de Estudos Avançados em Economia } \\
\text { Aplicada }\end{array}$ & SP - Brasil \\
\hline Angélica Toffano Seidel Calazans & Universidade de Brasília & DF - Brasil \\
\hline Antônio Luiz Rocha Dacorso & Universidade Federal de Sergipe & SE - Brasil \\
\hline Antonio Marcos de Queiroz & Universidade Federal de Goiás & GO - Brasil \\
\hline Antonio Zanin & Universidade Comunitária da Região de Chapecó & SC - Brasil \\
\hline Ariel Orlei Michaloski & Universidade Tecnológica Federal do Paraná & PR - Brasil \\
\hline Arlete Redivo & Universidade do Estado de Mato Grosso & MT - Brasil \\
\hline Armando Terribili Filho & Universidade Positivo & PR - Brasil \\
\hline Arno Paulo Schmitz & Universidade Federal do Paraná & PR - Brasil \\
\hline Boanerges do Amaral Couto & Universidade Federal Fluminense & RJ - Brasil \\
\hline Carlos Augusto de Oliveira & Universidade Federal Rural do Rio de Janeiro & RJ - Brasil \\
\hline Carlos Henrique Marques da Rocha & Universidade de Brasília & DF - Brasil \\
\hline Carlos Magri Ferreira & Empresa Brasileira de Pesquisa Agropecuária & GO - Brasil \\
\hline Carlos Roberto Martins & Empresa Brasileira de Pesquisa Agropecuária & RS - Brasil \\
\hline Cassiano Bragagnolo & Universidade Federal de São Carlos & SP - Brasil \\
\hline Célia Satiko Ishikiriyama & Pontifícia Universidade Católica do Rio de Janeiro & RJ - Brasil \\
\hline Celso Mitsuo Hino & Universidade de São Paulo & SP - Brasil \\
\hline Claudimar Pereira da Veiga & Universidade Federal do Paraná & PR - Brasil \\
\hline Cláudio Luís Carvalho Larieira & $\begin{array}{l}\text { Escola de Administração de Empresas de São } \\
\text { Paulo }\end{array}$ & SP - Brasil \\
\hline Cleiton Stigger Perleberg & Universidade Federal de Pelotas & RS - Brasil \\
\hline Dimas Soares Júnior & Instituto Agronômico do Paraná & PR - Brasil \\
\hline Edenis Cesar de Oliveira & Universidade Federal de São Carlos & SP - Brasil \\
\hline Edson Pacheco Paladini & Universidade Federal de Santa Catarina & SC - Brasil \\
\hline Edson Pereira da Mota & Universidade de São Paulo & SP - Brasil \\
\hline Elizete Antunes Teixeira & Universidade Federal de São João Del-Rei & MG - Brasil \\
\hline Ellen de Souza Marquez & Universidade Estadual do Norte do Paraná & PR - Brasil \\
\hline Ércio Roberto Proença & $\begin{array}{l}\text { Universidade Estadual Paulista Júlio de Mesquita } \\
\text { Filho }\end{array}$ & SP - Brasil \\
\hline Eugenio Libreloto Stefanelo & Universidade Federal do Paraná & PR - Brasil \\
\hline Felipe Mussarelli & $\begin{array}{l}\text { Programa de Educação Continuada em Economia } \\
\text { e Gestão de Empresas }\end{array}$ & SP - Brasil \\
\hline Fernanda Cristina Pierre & Faculdade de Tecnologia de Botucatu & SP - Brasil \\
\hline
\end{tabular}




\begin{tabular}{|c|c|c|}
\hline Revisores ad hoc 2017 & Instituição & UF/País \\
\hline $\begin{array}{l}\text { Fernanda Maria Pinto Freitas Ramos } \\
\text { Ferreira }\end{array}$ & Faculdade de Tecnologia de São Paulo & SP - Brasil \\
\hline Fernando dos Santos Sampaio & Universidade Estadual do Oeste do Paraná & PR - Brasil \\
\hline Flávio Reis dos Santos & Universidade Estadual de Goiás & GO - Brasil \\
\hline Gean Lopes da Luz & Universidade Comunitária da Região de Chapecó & SC - Brasil \\
\hline Gerson Pech & Universidade do Estado do Rio de Janeiro & RJ - Brasil \\
\hline Gilmara Bruschi Santos & $\begin{array}{l}\text { Escola Superior de Agronomia de Paraguaçu } \\
\text { Paulista }\end{array}$ & SP - Brasil \\
\hline Glauber Eduardo de Oliveira Santos & Universidade de São Paulo & SP - Brasil \\
\hline Haroldo José Torres da Silva & $\begin{array}{l}\text { Programa de Educação Continuada em Economia } \\
\text { e Gestão de Empresas }\end{array}$ & SP - Brasil \\
\hline Hélio Gomes de Carvalho & Universidade Tecnológica Federal do Paraná & PR - Brasil \\
\hline Hildo Meirelles de Souza Filho & Universidade Federal de São Carlos & SP - Brasil \\
\hline Inês Hexsel Grochau & Universidade Federal do Rio Grande do Sul & RS - Brasil \\
\hline Jairo Costa Fernandes & $\begin{array}{l}\text { Instituto Federal de Educação, Ciência e } \\
\text { Tecnologia Baiano }\end{array}$ & BA - Brasil \\
\hline Jakes Halan de Queiroz Costa & Universidade Federal de Alagoas & AL - Brasil \\
\hline Janderson Damaceno dos Reis & Universidade Federal de Viçosa & MG - Brasil \\
\hline João Batista de Camargo Junior & Universidade Metodista de Piracicaba & SP - Brasil \\
\hline João Luís Guilherme Benassi & Instituto Federal de São Paulo & SP - Brasil \\
\hline Jean Menossi & $\begin{array}{l}\text { Instituto Federal de Educação, Ciência e } \\
\text { Tecnologia do Sul de Minas Gerais }\end{array}$ & MG - Brasil \\
\hline Jeronimo Alves dos Santos & Universidade Federal de São Carlos & SP - Brasil \\
\hline Jose Nildo Tabosa & Instituto Agronômico de Pernambuco & PE - Brasil \\
\hline José Roberto Campos & $\begin{array}{l}\text { Instituto Federal do Mato Grosso do Sul -Três } \\
\text { Lagoas }\end{array}$ & MS - Brasil \\
\hline Juliana Lobo Paes & Universidade Federal Rural do Rio de Janeiro & RJ - Brasil \\
\hline Júlio Graeff Erpen & Universidade Federal de Santa Catarina & SC - Brasil \\
\hline Junior Ruiz Garcia & Universidade Federal do Paraná & PR - Brasil \\
\hline Karina Gomes de Assis & Universidade Federal de São Carlos & SP - Brasil \\
\hline Klaus de Oliveira Abdala & Universidade Federal de Goiás & GO - Brasil \\
\hline Leonardo Caixeta de Castro Maia & Universidade Federal de Uberlândia & MG - Brasil \\
\hline Leonardo de Melo Menezes & Universidade Estadual do Rio Grande do Sul & RS - Brasil \\
\hline Leonardo Ferraz Xavier & Universidade Federal Rural de Pernambuco & PE - Brasil \\
\hline Liane Werner & Universidade Federal do Rio Grande do Sul & RS - Brasil \\
\hline Lilian Maluf de Lima & Universidade de São Paulo & SP - Brasil \\
\hline Luciana Simões Rafagnin Marinho & Centro Universitário Dinâmica das Cataratas & PR - Brasil \\
\hline Luís Carlos Zucatto & Universidade Federal de Santa Maria & RS - Brasil \\
\hline Luzenira Alves Brasileiro & Universidade Estadual Paulista & SP - Brasil \\
\hline Marcelo Fodra & Universidade Federal de Uberlândia & MG - Brasil \\
\hline Márcia Valéria Paixão & $\begin{array}{l}\text { Instituto Federal de Educação, Ciência e } \\
\text { Tecnologia do Paraná }\end{array}$ & PR - Brasil \\
\hline Marcos Antonio Silva & Centro Universitário Carioca & RJ - Brasil \\
\hline Marcos Milan & Universidade de São Paulo & SP - Brasil \\
\hline Maria Antonia Ramos de Azevedo & Universidade Estadual Paulista & SP - Brasil \\
\hline Maria Aparecida Canale Balduino & Universidade Católica Dom Bosco & MS - Brasil \\
\hline Maria Cecília Perantoni Fuchs Ferraz & $\begin{array}{l}\text { Programa de Educação Continuada em Economia } \\
\text { e Gestão de Empresas }\end{array}$ & SP - Brasil \\
\hline Maria Cristina Bohnenberger & Universidade Feevale & RS - Brasil \\
\hline $\begin{array}{l}\text { Maria de Nasare Bona de Alencar } \\
\text { Araripe }\end{array}$ & Universidade Federal do Piauí & PI - Brasil \\
\hline Marta Cristina Marjotta-Maistro & Universidade Federal de São Carlos & SP - Brasil \\
\hline Matheus Dhein Dill & Universidade Federal Rural de Pernambuco & PE - Brasil \\
\hline Maura Seiko Tsutsui Esperancini & Universidade Estadual Paulista & SP - Brasil \\
\hline Nelson Cerino Franco Júnior & $\begin{array}{l}\text { Programa de Educação Continuada em Economia } \\
\text { e Gestão de Empresas }\end{array}$ & SP - Brasil \\
\hline Nilson Robson Guedes Silva & Centro de Educação Profissional de Campinas & SP - Brasil \\
\hline Odilon José de Oliveira Neto & Universidade Federal de Uberlândia & MG - Brasil \\
\hline
\end{tabular}




\begin{tabular}{|c|c|c|}
\hline Revisores ad hoc 2017 & Instituição & UF/País \\
\hline Orlando Celso Longo & Universidade Federal Fluminense & RJ - Brasil \\
\hline Patrik Luiz Pastori & Universidade Federal do Ceará & CE - Brasil \\
\hline Paulo Carteri Coradi & Universidade Federal de Santa Maria & RS - Brasil \\
\hline Paulo Dabdab Waquil & Universidade Federal do Rio Grande do Sul & RS - Brasil \\
\hline Pedro Henrique Melo Albuquerque & Universidade de Brasília & DF - Brasil \\
\hline Pedro Paiva & Universidade de São Paulo & SP - Brasil \\
\hline Priscila Fortes & $\begin{array}{l}\text { Programa de Educação Continuada em Economia } \\
\text { e Gestão de Empresas }\end{array}$ & SP - Brasil \\
\hline Rafael Simões Coelho Barone & $\begin{array}{l}\text { Programa de Educação Continuada em Economia } \\
\text { e Gestão de Empresas }\end{array}$ & SP - Brasil \\
\hline Raquel Castellucci Caruso Sachs & Agência Paulista de Tecnologia dos Agronegócios & SP - Brasil \\
\hline Ricardo Carneiro Brumatti & Universidade Federal de Mato Grosso do Sul & MS - Brasil \\
\hline Ricardo Coser Mergulhão & Universidade Federal de São Carlos & SP - Brasil \\
\hline Ricardo Cruvinel Dornelas & Universidade Federal de Goiás & GO - Brasil \\
\hline Ricardo Harbs & Universidade de São Paulo & SP - Brasil \\
\hline Ricardo Shirota & Universidade de São Paulo & SP - Brasil \\
\hline Roberto Antonio Colenci & Faculdade de Tecnologia de Botucatu & SP - Brasil \\
\hline Roberto Arruda de Souza Lima & Universidade de São Paulo & SP - Brasil \\
\hline Rodrigo Anselmo Tarsitano & Universidade do Estado de Mato Grosso & MT - Brasil \\
\hline Romano Timofeiczyk Junior & Universidade Federal do Paraná & PR - Brasil \\
\hline Ronaldo Rosa dos Santos Junior & Faculdade Araguaia & GO - Brasil \\
\hline $\begin{array}{l}\text { Rosária de Fátima Segger Macri } \\
\text { Russo }\end{array}$ & Universidade Nove de Julho & SP - Brasil \\
\hline Rubens Augusto de Miranda & Empresa Brasileira de Pesquisa Agropecuária & MG - Brasil \\
\hline Sandra Cristina de Moura Bonjour & Universidade Federal de Mato Grosso & MT - Brasil \\
\hline Sara Martins Vieira Zimmermann & Universidade de São Paulo & SP - Brasil \\
\hline Silvio Campos Guimarães & Fundação Getulio Vargas & SP - Brasil \\
\hline Sydney Manzione Junior & Escola Superior de Propaganda e Marketing & SP - Brasil \\
\hline Tamara Esteves de Oliveira & Universidade Federal do Rio Grande do Sul & RS - Brasil \\
\hline Terezinha Bezerra Albino Oliveira & Universidade Federal de Alagoas & AL - Brasil \\
\hline Thiago Bernardino de Carvalho & $\begin{array}{l}\text { Centro De Estudos Avançados Em Economia } \\
\text { Aplicada - USP }\end{array}$ & SP - Brasil \\
\hline $\begin{array}{l}\text { Vera Lucia de Faveri Fernandes e } \\
\text { Silva }\end{array}$ & Centro Universitário Claretiano de Batatais & SP - Brasil \\
\hline Vera Lúcia Jacob Chaves & Universidade Federal do Pará & PA - Brasil \\
\hline Vitor Pires Vencovsky & Faculdade de Tecnologia de Piracicaba & SP - Brasil \\
\hline Wagner Vicente Pereira & Universidade Federal do Paraná & PR - Brasil \\
\hline Wilson Valente da Costa Neto & Universidade Federal do Pampa & RS - Brasil \\
\hline
\end{tabular}

